\title{
Der Kreis um Hans Kelsen in Lateinamerika Wie die Reine Rechtslehre Lateinamerika eroberte*
}

\begin{abstract}
Hans Kelsen, one of the most important legal theorists of the 20th century, was and in fact still is as popular in Latin America as he is in Europe. The article examines Kelsen's reception in the Spanish speaking world, especially his reception by the legal philosopher Carlos Cossio and gives an overview of Kelsen's visits to Latin America, namely to Cuba in 1941, to Brazil, Uruguay and Argentina in 1949 and to Mexico in 1960.
\end{abstract}

\section{Einleitung}

Ein wesentlicher Grund für den Einfluss, den die von Hans Kelsen entwickelte „Reine Rechtslehre" weltweit gewinnen konnte, war der Umstand, dass Kelsen stets schulenbildend wirkte, d.h. bemüht war, seine Theorien und Methoden an zumeist jüngere Kolleginnen und Kollegen weiterzugeben. Vermutlich schon 1913 begann er, in seiner Wohnung im VIII. Wiener Gemeindebezirk in der Wickenburggasse 23 Privatseminare abzuhalten, woraus die „Wiener rechtstheoretische Schule“ entstand. Aber auch während seiner Tätigkeit in Köln (1930-1933), Genf (1933-1940) und in den Vereinigten Staaten (ab 1940) trachtete Kelsen nicht nur danach, den Kontakt zu seinen alten Schülerinnen und Schülern zumindest im Briefweg aufrecht zu erhalten, sondern immer wieder auch neue Schülerinnen und Schüler für seine Lehre zu gewinnen. So entstand ein weltumspannendes Netzwerk von Wissenschafterinnen und Wissenschaftern, die die Reine Rechtslehre direkt von ihrem Begründer kennen gelernt hatten und wenigstens zum Teil - und auf sehr unterschiedliche Art und Weise - fortführten und weiterentwickelten.

2008 veröffentlichte das Wiener Hans KelsenInstitut einen Sammelband, betitelt „Der Kreis um Hans Kelsen. Die Anfangsjahre der Reinen
Rechtslehre“. Der Untertitel erklärt sich aus dem Umstand, dass die 28 in diesem Band porträtierten Personen allesamt mit Kelsen noch vor dessen Flucht aus Europa 1940 „in persönlichen Kontakt gekommen waren ". ${ }^{1}$ Die Kontakte, die Kelsen nach seiner Emigration in die Vereinigten Staaten neu knüpfen konnte, blieben also ausgeklammert; ihre Recherche ist von Europa aus weit aufwändiger als jene zu den Anfangsjahren.

In diesem Sinne möchte die gegenständliche Arbeit einen ersten Überblick über die persönlichen Kontakte Hans Kelsens zu lateinamerikanischen Rechtswissenschaftern geben und darstel-

\footnotetext{
* Der Artikel enthält Ergebnisse des FWF-Projekts P 23747 „Hans Kelsens Leben in Amerika (1940-1973) und die weltweite Verbreitung seiner Rechtslehre". Im Rahmen dieses Projekts hatte die Autorin des gegenständlichen Beitrages u.a. die Aufgabe, die Beziehungen Kelsens zu lateinamerikanischen Juristen zu untersuchen und unternahm 2012 gemeinsam mit dem Projektleiter, Prof. Thomas Olechowski, zwei Amerika-Reisen (Argentinien, Brasilien, Mexiko, Uruguay, USA), bei denen eine Reihe von Interviews mit Zeitzeugen geführt wurden, worauf an entsprechender Stelle noch gesondert hingewiesen werden wird. Gedankt sei Frau Dr. Tamara Ehs für das Zurverfügungstellen ihrer Rechercheergebnisse im Rahmen des genannten Projektes, namentlich zu Kelsens Beziehungen zur Rockefeller Foundation.

${ }^{1}$ WALTER, JABLONER, ZELENY, Einleitung 5.
} 
len, inwieweit diese bemüht waren, die Reine Rechtslehre in ihren Ländern fortzuführen und weiterzuentwickeln. Im Zentrum stehen dabei Kelsens Lateinamerika-Reisen 1941, 1949 und 1960 sowie insbesondere die Auseinandersetzung mit dem argentinischen Juristen Carlos Cossio, der zwar schon bald ein von Kelsen deutlich abweichendes Theorem, die sog. Egologische Rechtslehre, entwickelte, der aber vielleicht gerade dadurch wesentlich $\mathrm{zu}$ der bis heute ungebrochenen Popularität Kelsens in Lateinamerika beigetragen hat.

\section{Erste Kontakte in die spanischsprachige Welt}

Hans Kelsens Kontakte nach Südamerika bestanden ab Mitte der 1920er Jahre. So verfasste Hans Kelsen 1926 einige „Bemerkungen zur Chilenischen Verfassung", ${ }^{2}$ wobei heute unklar ist wie es dazu kam. Manchen südamerikanischen Kelsenforschern zufolge ${ }^{3}$ könnte der chilenische Staatspräsident Aturo Alessandri, der von September 1924 bis März 1925 in Frankreich, der Schweiz und Italien im Exil war, ${ }^{4}$ Kelsen mit einem Gutachten über die chilenische Verfassung von 1925 beauftragt haben, wofür es allerdings keinerlei Hinweise gibt. Auch ist über eventuell aus Chile stammende Schüler bzw. sonstige Kontakte Kelsens nach Chile während seiner Wiener Zeit nichts bekannt.

Als Kelsens „Eintrittskarte“ in die spanischsprachige Welt kann wohl der ursprünglich aus Guatemala stammende Jurist Luis Recasens Siches bezeichnet werden:

Luis Recasens Siches, 1903 in Guatemala geboren, hatte in Barcelona Rechtswissenschaften studiert und kam im Zuge einer Reihe von For-

\footnotetext{
${ }^{2}$ Kelsen, Bemerkungen zur Chilenischen Verfassung. ${ }^{3}$ So z.B. Augustin Squella von der Universität von Valparaiso.

${ }^{4}$ COLLIER, SATER, History of Chile 211.
}

schungsaufenthalten (u.a. bei José Ortega y Gasset $^{5}$ in Madrid, bei Rudolf Stammler und Hermann Heller in Berlin und bei Giorgio del Vecchio in Rom) von November 1926 bis April 1927 nach Wien, um Hans Kelsens Vorlesungen zu hören. ${ }^{6}$ Er nahm an Kelsens Privatseminaren in der Wickenburggasse teil, wo er auch Kelsens Schüler Rudolf A. Métall und Josef L. Kunz - die beide spanisch sprachen - kennengelernt haben wird.7 Schon während Recasens Siches Aufenthalt in Wien entwickelte sich eine enge Freundschaft zwischen Métall, Kunz und Recasens Siches, die später bei der Verbreitung von Kelsens Reiner Rechtslehre in der spanischsprachigen Welt eine große Rolle spielen sollte. Recasens Siches war in Kelsens Vorlesungen allerdings keineswegs der einzige Student aus der spanischsprachigen Welt gewesen, denn zeitgleich mit Recasens Siches war auch der Kubaner Antonio Sanchez de Bustamante y Montoro zu Kelsen nach Wien gekommen, um Kelsens Vorlesungen $\mathrm{zu}$ hören. ${ }^{8}$ Über Bustamante kam später der Kontakt zwischen Kelsen und dem in Kuba lebenden spanischen Rechtsphilosophen Emilio Fernandez Camus zustande, zu dessen Werk „Gegenwärtige Rechtsphilosophie” Kelsen 1932 ein Vorwort verfasste. ${ }^{9}$

\footnotetext{
${ }^{5}$ Siehe dazu Anm. 28.

${ }^{6}$ "Durante el período que cubrieron los meses de noviembre de 1926 a abril de 1927 se trasladó a la Universidad de Viena. En dicha institución estudió Teoría del Derecho, Filosofía, Teoría General del Estado y Sociología, becado por el Ministerio de Instrucción Pública de España, a propuesta de la Junta para la Ampliación de Estudios e Investigaciones Científicas, por Real Orden de 19 de junio de 1926 (Gaceta del 31 de julio). Estuvo bajo la dirección de los profesores Hans Kelsen, Fritz Schreier, Félix Kaufmann y R. Reininger. También acudió a las lecciones de los profesores Bühler y Adler."

(http://www.uc3m.es/portal/page/portal/instituto_fig uerola/programas/phu/diccionariodecatedraticos/ lcatedraticos/rsiches - abgerufen am 08. 08. 2013). So NOGUEIRA DiAs, Recaséns Siches 366.

${ }^{7}$ MÉTALL, Hans Kelsen y su escuela 23.

${ }^{8}$ Ebd.

${ }^{9}$ FERnÁndez CAMUS, Filosofia juridica 5-8.
} 
1928 kehrte Bustamante y Montoro nach Kuba und Recasens Siches nach Spanien zurück, wo letztgenannter sich dem Kreis um José Ortega y Gasset anschloss und gemeinsam mit Justino de Azcárate Flores Hans Kelsens „Grundriß einer allgemeinen Theorie des Staates" ins Spanische zu übersetzen begann. ${ }^{10}$ Nach kurzen Stationen an den Universitäten von Santiago de Compostela, Valladolid und Salamanca, erhielt Recasens Siches schließlich eine Professur in Madrid, ${ }^{11}$ wo unter anderem der Spanier Luis Legaz y Lacambra bei ihm studierte. Auf Empfehlung Recasens Siches' kam Legaz y Lacambra im Studienjahr 1929/30 zu Kelsen nach Wien ${ }^{12}$ und hörte dort neben Kelsens Vorlesungen zur Staatstheorie auch Vorlesungen bei Alfred Verdross, Fritz Schreier und Felix Kaufmann. ${ }^{13}$ Wieder in Spanien, begann Legaz y Lacambra mehrere Artikel über die Wiener Schule im Allgemeinen und Kelsens Rechtstheorie im Besonderen zu publizieren. Auch in seiner Dissertation, zu der Recasens Siches das Vorwort verfasste, setzte sich Legas y Lacambra kritisch mit Kelsen und der Wiener Rechtstheoretischen Schule auseinander. ${ }^{14}$

1933 - also noch vor Erscheinen der ersten Auflage der Reinen Rechtslehre im deutschsprachigen Raum - verfasste Luis Legaz y Lacambra unter dem Titel „Reine Rechtslehre. Methode und fundamentale Konzepte“ eine Teilübersetzung von Kelsens „Reiner Rechtslehre“ ins Spanische. ${ }^{15} 1934$ folgte eine Übersetzung von Kelsens „Allgemeine Staatslehre“ ins Spanische

\footnotetext{
${ }^{10}$ KeLSEN, Compendio esquemático; Vgl. (http://www.uc3m.es/portal/page/portal/instituto_fig uerola/programas/phu/diccionariodecatedraticos/ lcatedraticos/rsiches - abgerufen am 08. 08. 2013)

${ }^{11}$ NogUeIRA DiAs, Recasens Siches 366.

12 Siehe dazu Anm. 8 bzw. Robles Morchon, Luis Legaz y Lacambra.

${ }^{13}$ Ebd. (siehe Anm. 13).

${ }^{14}$ LegaS Y LACAMBRA, Kelsen.

${ }^{15}$ Kelsen, Teoria Pura del Derecho.
}

durch Lacambra, ${ }^{16}$ womit Kelsens Eintritt in die spanische rechtstheoretische Landschaft als vollzogen betrachtet werden kann.

Ebenfalls 1934 wurde Hans Kelsen, wohl über die Kontakte seines ehemaligen Schülers und Assistenten Rudolf A. Métall, von der brasilianischen Nationalversammlung mit der Erstellung eines Gutachtens über die Kompetenz der konstituierenden Nationalversammlung beauftragt. ${ }^{17}$

$\mathrm{Ab}$ den frühen 1930er Jahren, als die politische Lage in Spanien in Folge eines (misslungenen) Militärputschs immer unbeständiger wurde und Spanien unausweichlich auf einen Bürgerkrieg zusteuerte, kam es zu einer Immigrationswelle spanischer Rechtsgelehrter und Intellektueller nach Südamerika. Diese hatte zur Folge, dass die neuesten europäischen (rechts-)philosophischen Entwicklungen nach Südamerika „importiert” wurden. Auf diese Weise hielt wohl auch die Reine Rechtslehre Kelsens Einzug in viele südamerikanische Universitäten. Unter den spanischen Immigranten, die Spanien im Zuge des spanischen Bürgerkrieges verließen, war auch Luis Recasens Siches. 1937 erhielt Recasens Siches eine Professur an der Universität von Mexiko (UNAM) ${ }^{18}$ und sollte dort in den folgenden Jahrzehnten einen wesentlichen Teil zur Verbreitung der Reinen Rechtslehre beitragen.

Auch in Argentinien begann sich die Reine Rechtslehre durch die spanischen Immigranten rasch zu verbreiten. Anzunehmen ist, dass auf diese Weise auch ein damals noch nahezu unbekannter junger argentinischer Jurist namens Carlos Cossio auf Kelsens Rechtslehre aufmerksam wurde.

\footnotetext{
${ }^{16}$ KELSEN, Teoría general del Estado.

${ }^{17}$ KelsEN, A competência da Assembléia Nacional Constituinte.

${ }^{18}$ NogueIRa Dias, Recasens Siches 366ff.
} 
1903 in der Provinz Tucuman im Norden Argentiniens geboren, studierte Cossio an der Universität von Buenos Aires Rechtswissenschaften, schloss sich noch während seines Studiums der studentischen Reformbewegung (movimiento reformista) an und verfasste 1927 auch seine Dissertation über diese. ${ }^{19} \mathrm{Ab} 1934$ Dozent an der Universidad Nacional von La Plata, nahm Cossio in seinem 1936 erschienenen Werk „Das reine Konzept der Revolution“20 erstmals explizit auf Kelsens Reine Rechtslehre Bezug. Genau in dieser Zeit begann sich auch Kelsens ehemaliger Schüler Josef Laurenz Kunz, der 1934 in die USA immigriert und dort eine Professur an der University of Toledo, Ohio, bekommen hatte, intensiv mit der damals gegenwärtigen lateinamerikanischen Rechtsphiliosophie zu beschäftigen. ${ }^{21}$ Zahlreiche Forschungsreisen führten Kunz ab 1937 unter anderem nach Argentinien, wo er auch Carlos Cossio kennenlernt haben dürfte. Nach eigenen Angaben stand Cossio ab 1937 - einem Zeitpunkt, der mit Josef Kunz' Argentinienaufenthalt zusammenfallen dürfte mit Kelsen in Briefkontakt und sandte diesem regelmäßig all jene seiner Arbeiten, in denen er auf Kelsen und dessen Reine Rechtslehre Bezug nahm. ${ }^{22}$ Am 7. November 1938 wurde Kelsen vom Instituto Argentino de Filosofia Juridica y Social, das im selben Jahr von Carlos Cossio gegründet worden war, zum Ehrenmitglied ernannt. ${ }^{23}$

Kelsen lebte zu dieser Zeit in Genf, wo sich auch der Sitz des Völkerbundes befand. In Genf knüpfte Kelsen Kontakte zu einigen südamerikanischen Diplomaten und Völkerrechtlern, wie zum kolumbianischen Generalkonsul J.M. Ye-

\footnotetext{
${ }^{19}$ Cossio, Reforma Universitaria.

${ }^{20}$ Cossio, Concepto puro.

${ }^{21}$ KAMMERHOFER, Josef Laurenz Kunz 246.

${ }^{22}$ Cossio, Como ve Kelsen.

${ }^{23}$ MÉTALL, Kelsen 99; so auch SARLO, Gira sudamericana 406.
}

pes $^{24}$ oder zum argentinischen Völkerrechtler Luis Podesta Costa, ${ }^{25}$ die sich schließlich, als Kelsen 1940 Europa im Zuge des 2. Weltkrieges verlassen musste, bemühten, Kelsen ein Visum in ein lateinamerikanisches Land $\mathrm{zu}$ verschaffen. ${ }^{26}$ Im sprichwörtlich letzten Moment gelang es Kelsen jedoch, über die New School for Social Research ein ex-quota Visum, welches ihn zur Einreise in die USA berechtigte, zu erhalten und so verließ Kelsen gemeinsam mit seiner Gattin Grete am 28. Mai 1940 Genf, um in den USA ein neues Leben zu beginnen. Das akademische Jahr 1940/41 verbrachte Kelsen an der Harvard Law School wo er eine auf ein Jahr beschränkte Lectureship erhielt, die von der Rockefeller Foundation teilfinanziert wurde. ${ }^{27}$

Mit Ausbruch des 2. Weltkriegs in Europa, der viele, vor allem jüdischstämmige Wissenschaftler dazu zwang, Europa zu verlassen und sich in Nord- und Südamerika eine neue Existenz aufzubauen, kam es in Südamerika zu einem neuen Einwanderungsschub europäischer (Rechts-)

philosophen. So begab sich der spanische Philosoph José Ortega y Gasset² 1939 nach Argentinien ins Exil, wo er auf eine ganze Generation junger Juristen wie Jaime Perriaux großen Einfluss ausüben sollte. Neben renommierten Juris-

\footnotetext{
${ }^{24}$ MÉTALL, Kelsen 76.

${ }^{25}$ Interview CAMINOS.

${ }^{26}$ MÉTALL, Kelsen 76.

${ }^{27}$ MÉTALl, Kelsen 77.

28 José Ortega y Gasset (1883-1955), Hauptvertreter des spanischen "Perspektivismus", verließ während des spanischen Bürgerkriegs Spanien und begab sich zuerst nach Paris, später nach Holland und schließlich bis 1942 nach Argentinien ins Exil. Der (spanische) „Perspektivismus" geht in Anlehnung an Gottfried Wilhelm Leibniz und Friedrich Nietzsche davon aus, dass die Wirklichkeit nicht objektiv feststeht, sondern je nach Perspektive des betrachtenden Individuums variieren kann. Das menschliche Denken, Erkennen und Handeln unterliegt zahlreichen Beschränkungen, die sich aus Zeit und Raum oder anderen Bedingungen ergeben.
} 
ten und (Rechts-) philosohen wie dem Spanier Ortega y Gasset oder dem deutschen Prozessrechtler James Goldschmidt, ${ }^{29}$ bis 1934 Professor für Verwaltungsrecht an der FriedrichWilhelms-Universität Berlin, immigrierten auch einige Kelsen-Schüler nach Südamerika, unter ihnen Rudolf A. Metall ${ }^{30}$ und Hans Klinghoffer, ${ }^{31}$ die sich 1940 bzw. 1941 in Rio de Janeiro, Brasilien niederließen. Otto E. Langfelder, der um 1929 bei Kelsen und Felix Kaufmann in Wien studiert hatte, immigrierte 1941 nach Buenos Aires. Dort schloss er sich dem Kreis um den argentinischen Rechtsphilosophen Carlos Cossio an, zu dem damals auch Rechtstheoretiker wie Ambrosio Lucas Gioja, Julio Cesar Cueto Rua, Genaro Carrió, José Vilanove, Enrique Aftalión und Abel Aristegui angehörten. ${ }^{32}$ Ebenfalls nach Argentinien gelangt war ein gewisser Rodolfo Bledel, den Kelsen 1941 mit der spanischen Übersetzung seines Aufsatzes „The Pure Theory of Law and Analytical Jurisprudence"33 beauftragte, welche noch im selben Jahr in der argentinischen Zeitschrift „La Ley" erschien. ${ }^{34}$

Zeitgleich mit Langfelders Eintreffen in Argentinien erschien 1941 die erste vollständige spanische Übersetzung der Reinen Rechtslehre, zu der Carlos Cossio das Vorwort verfasste. ${ }^{35} \mathrm{Ob}$ wohl Cossio Kelsen im Vorwort geradezu Rosen streute und ihm seinen Lob und seine Anerkennung aussprach, fiel dieses, liest man es genauer, durchaus kritisch aus und enthielt bereits erste "Anzeichen" für jene von Cossio vorgenommene Interpretation der Reinen Rechtslehre, die später als egologische Auslegung der Reinen

\footnotetext{
${ }^{29}$ http://de.wikipedia.org/wiki/James_Goldschmidt (abgerufen am 08. 08. 2013).

${ }^{30}$ BERSIER LADAVAC, Rudolf Aladár Métall 316.

${ }^{31}$ KLEIN, Hans (Itzhak) Klinghoffer 176.

32 Pettoruti, Verbindung zwischen Wien und La Plata 232.

${ }^{33}$ KELSEN, Pure Theory of Law.

${ }^{34}$ KeLSEN, Teoria pura del derecho.

${ }^{35}$ Cossio, Prólogo.
}

Rechtslehre bekannt werden - und Kelsen einige harte Auseinandersetzungen - bereiten sollte.

\section{Kelsens erste Reise nach Lateinamerika}

Kelsens erste Reise nach Lateinamerika führte ihn im November 1941 nach Kuba, wo er von 15. bis 22. November 1941 in Havanna an der Second American Conference of National Committees on Intellectual Cooperation teilnahm. Die Kosten von Kelsens Kuba Reise wurden von der Rockefeller Foundation $^{36}$ übernommen, die sich insbesondere zu Beginn der 1940er Jahre um eine Vertiefung der kulturelle Beziehung zu Lateinamerika bemühte. ${ }^{37}$ Auf Kuba kam es zu einem Wiedersehen Kelsens mit seinen ehemaligen Schülern Antonio Sanchez de Bustamante y Montoro, mittlerweile Professor für Rechtsphilosophie an der Universität von Havanna und Luis Recasens Siches, mittlerweile an der UNAM in Mexiko, der in Begleitung seines mexikanischen Kollegen Eduardo Garcia Maynez nach Kuba gereist war. ${ }^{38}$ Zwar hatte Garcia Maynez 1932 und 1933 gemeinsam mit seinem Kollegen Mario de la Cueva Studienaufenthalte in Wien und Berlin ${ }^{39}$ verbracht, doch lernte er Hans Kelsen erst auf 1941 auf Kuba persönlich kennen. ${ }^{40}$

Auch den auf Kuba lebenden spanischen (Rechts-) Philosophen Emilio Fernandez Camus, mit dem Kelsen seit den frühen 30er Jahren in

\footnotetext{
${ }^{36}$ Rockefeller Archive Center, Collection RF, Record Group 1.1, Series 2003, Box 344, Folder 4089.

${ }^{37}$ LÜBKEN, Bedrohliche Nähe 112.

38, ,.... Me refiero a una conversación que hace algunos años tuvimos con Kelsen, Antonio Sánchez de Bustamante y Montoro, Emilio Fernández Camus y yo, en la residencia del segundo": GARCíA MAYNEZ, Introducción 9.

${ }^{39}$ RodRIgUeZ EsPINOZA, Quien fue Eduardo Garcia Maynez? http://www.hectorrodriguezespinoza.com/noticias.ph p?categoria=110 (abgerufen am 08. 08. 2013).

${ }^{40}$ Siehe dazu: Eduardo Garcia Maynez an Hans Kelsen, 10. 1. 1947, HKI, Nachlass Kelsen 15r.58.
} 
Kontakt stand, traf Kelsen 1941 im Rahmen der besagten Konferenz zum ersten Mal persönlich. Während Kelsens Aufenthalt in Kuba, dürfte unter anderem über Carlos Cossio und dessen beginnende egologische Auslegung der Reinen Rechtslehre gesprochen worden sein, zumal Bustamante y Montoro (offensichtlich in einem Brief vom 28. November 1841) Cossio berichtete, Kelsen habe ihm auf Kuba die Frage gestellt, wie er der egologischen Interpretation der Reinen Rechtslehre gegenüberstünde. Als er sich mit der egologischen Auslegung d'accord zeigte, sei Kelsen wütend geworden und habe betont, dass diese insofern unrichtig sei, als es ein und dieselbe Logik - und nicht eine eigene juristische Logik - sei, die allen Wissenschaften zugrunde liege. ${ }^{41}$

Nach dem Treffen auf Kuba luden Recasens Siches und Garcia Maynez sowie der damalige Dekan der juristischen Fakultät der UNAM Alfonso Noriega Kelsen nach Mexiko ein, wo er im Jänner 1943 einige Vorträge halten sollte. ${ }^{42}$ Wie sich aus der Korrespondenz Kelsens ergibt, konnte er diese Reise aber aufgrund „administrativer Schwierigkeiten" (anscheinend weil er 1943 noch nicht US-Staatsbürger war) nicht antreten. ${ }^{43}$ Die bereits vorbereiteten Vorträge wurden von Kelsen jedoch nach Mexiko gesandt und dort in der Übersetzung von Eduardo Garcia Maynez publiziert. Ebenso übersetzte Maynez 1943 mit einer Einleitung von Alfonso Noriega Kelsens „Der innerstaatliche Vertrag und der völkerrechtliche Vertrag"44 ins Spanische und publizierte es in Mexiko.

In der Revista de la Escuela Nacional de Jurisprudencia wurde in der letzten Ausgabe des Jahres

\footnotetext{
${ }^{41}$ Cossio, Como ve Kelsen 1.

42 FLORES, Una visita a Hans Kelsen 3.

${ }^{43}$ Hans Kelsen an Eduardo Garcia Maynez, 22. 7. 1946, HKI, Nachlass Kelsen 15r.58; Luis Recasens Siches an Hans Kelsen, 28. 5. 1946, HKI, Nachlass Kelsen 15r.58.

${ }^{44}$ KeLSEN, Contracto y el tratado.
}

1946 neuerlich Kelsens Kommen angekündigt. ${ }^{45}$ Aber auch dieser Einladung Garcia Maynez', infolge deren Kelsen zwischen 9. und 22. Februar 1947 an der UNAM Vorträge über „auserwählte Probleme der Reinen Rechtslehre“46 (genauer gesagt auf Wunsch des Dekans der rechtswissenschaftlichen Universität Virgilio Dominguez über "The Metamorphoses of the Idea of Justice" 47 auf französisch, sowie drei Vorträge über die Charter der UN am Instituto Mexicano de Economia in englischer Sprache) halten sollte ${ }^{48}$ konnte Kelsen keine Folge leisten. In letzter Minute sagte er seine Mexikoreise aufgrund von gesundheitlichen Problemen ab. ${ }^{49}$

\section{Kelsens Cossio zu verdankende wachsende Popularität in Lateinamerika}

Am 12. Oktober 1941 veröffentlichte Carlos Cossio, anlässlich von Kelsens 60. Geburtstag, einen Artikel, in dem er Kelsen als den größten Juristen der Gegenwart bezeichnete. ${ }^{50}$ Von da an begann sich Cossio jedoch mehr und mehr von Kelsen abzuwenden: Der Hauptvorwurf Cossios bestand zunehmend darin, dass Kelsen die Existenz einer eigenen Logik, die den Rechtswissenschaften zugrunde liege, grundlos ablehne. 1944 war der Bruch zwischen Reiner und Egologischer Rechtslehre endgültig vollzogen, als Cossio sein Buch „Die egologische Rechtslehre und

\footnotetext{
${ }^{45}$ Revista de la Escuela Nacional de Jurisprudencia, Nr. 32 (octubre-diciembre 1946) 373.

${ }^{46}$ Hans Kelsen an Eduardo Garcia Maynez, 22. 7. 1946, HKI, Nachlass Kelsen 15r.58.

${ }^{47}$ Revista de la Escuela Nacional de Jurisprudencia, Nr. 44 (octubre-diciembre 1949) 83-123.

${ }^{48}$ Hans Kelsen an Eduardo Garcia Maynez vom 22. 7. 1946, HKI, Nachlass Kelsen 15r.58.

${ }^{49}$ Hans Kelsen an Garcia Maynez, undatiert, HKI, Nachlass Kelsen 15r.58.

${ }^{50}$ Cossio, Jurista de la epoca contemporanea.
} 
das Konzept der Freiheit", ${ }^{51}$ zu dem Kelsens ehemaliger Schüler Otto E. Langfelder das Vorwort verfasste, publizierte. Zwar sah Cossio in diesem Buch die egologische Rechtslehre weiterhin als bloße Interpretation und kritische Ergänzung der Reinen Rechtslehre an, in Wirklichkeit hatte ging es ihm jedoch um etwas mehr: Er analysierte die Verbindung zwischen Norm und Verhalten und machte dabei das Verhalten primär zum Gegenstand der Rechtswissenschaften. ${ }^{52}$ Fast entschuldigend, so, als hätte Langfelder ein schlechtes Gewissen, das Vorwort $\mathrm{zu}$ Cossios Werk verfasst zu haben, schrieb dieser an Kelsen, dass er, obwohl er das Vorwort zu Cossios Egologie verfasst habe, "nicht immer mit ihm [Cossio] gehe. ${ }^{453}$

Die eigene Gestalt, die Kelsens Reine Rechtslehre durch die etwas eigenwillige Interpretation Cossios im spanischsprachigen Raum anzunehmen drohte, wurde von Hans Kelsen von Berkeley aus, wo er seit 1942 am Political Science Department lehrte, gespannt verfolgt. Das Hauptproblem dabei war, dass Kelsen kein Spanisch sprach und sich somit sein eigenes Urteil über die Egologie Cossios nur mit Hilfe Dritter bilden konnte. In diesem Sinne kam vor allem Kelsens ehemaligem Schüler Josef Kunz eine wichtige Rolle zu. 1942 war er von der Association of American Law Schools mit einer Untersuchung der gegenwärtigen lateinamerikanischen Rechtsphilosophie beauftragt worden $^{54}$ und hatte sich im Rahmen dieser Untersuchungen ausgiebig mit der Egologie Cossios auseinandergesetzt. Anlässlich seiner Buchbesprechung zu Cossios „Die egologische Rechtslehre und

\footnotetext{
${ }^{51}$ Cossio, Teoría egológica.

52 GASSNER, OlECHOWSKI, Egologische Rechtslehre 6.

${ }^{53}$ Otto Langfelder an Hans Kelsen, 20. 12. 1947, HKI, Nachlass Kelsen: 16b2. 59.

${ }^{54}$ Das Ergebnis von Kunz Recherchen waren drei Teilberichte, die im Jahr 1949 in der April-, Juli- und Oktober-Ausgabe der New York Law Quarterly Review veröffentlicht wurden; gesammelt in: KUNZ, LatinAmerican philosophy.
}

das Konzept der Freiheit", schrieb Kunz am 15. Mai 1945 einen Brief an Kelsen und kam dabei zu folgenden Schluss:

„[...] Ich glaube, dass Cossio Ihre Rechtslehre richtig entfaltet und sie haben ihm in einem Brief die Orthodoxie seiner Auffassung bestätigt. (Nur ist nach ihm der Rechtssatz ein disjunktives, nicht ein hypothetisches Urteil) Aber: er reduziert Ihre Reine Rechtslehre zu nackter formaler Rechtslogik. Sie ist daher keine vollständige Rechtstheorie, denn sie ist nach ihm nur im geringen Grade transzendentale Rechtslogik. Sie ist keine Rechtsphilosophie im metaphysischen Sinn, sie ist aber auch nicht Rechtswissenschaft.“ Abschließend meinte Kunz: „[...] Das alles klingt ganz nett. Cossio bleibt innerhalb der Positivität und vermeidet im Gegensatz zu Garcia Maynez und Recanses Siches den Rekurs in die Metaphysik. [...] Das gefährlichste an Cossio ist aber, dass er Recht dem menschlichen Verhalten gleichsetzt und so in die Soziologie abdriftet. “ 55

Kelsens Antwortschreiben an Kunz ist nicht erhalten, doch scheint er dessen Analyse der egologischen Rechtslehre zuzustimmen, zumal Kunz in seinem Brief vom 19. Juni 1945 aus Cambridge an Kelsen schreibt: „[...] Ihre Zeilen bestärken mich in meiner Skepsis gegenüber der Grundlage von Cossios Theorie.“56

Aber auch in Argentinien wurden Kunz' und Kelsens Reaktionen auf Cossios Werk genau verfolgt: So äußerte sich Kelsens ehemaliger Schüler Otto E. Langfelder, der mittlerweile durch Cossios Unterstützungen zum Dozenten an der Universität von Buenos Aires aufgestiegen war, in einem Brief an Kelsen zu Kunz' Rezession. Langfelder schrieb, Kunz habe die geistige Situation hier richtig, aber doch etwas zu pessimistisch geschildert, denn immerhin habe

\footnotetext{
${ }^{55}$ Josef Kunz an Hans Kelsen, 15. 5. 1945, HKI, Nachlass Kelsen 16b1.59.

${ }^{56}$ Josef Kunz an Hans Kelsen, 16. 6. 1945, HKI, Nachlass Kelsen 16b1.59.
} 
der starke Druck Cossios dazu geführt, dass Kelsens Name nicht mehr mit der Etikette Formalismus behaftete sei. ${ }^{57}$

Im Zuge der United Nations Conference on International Organization, die vom 25. April 1945 bis zum 26. Juni 1945 in San Francisco stattfand, um die Charta der Vereinten Nationen auszuarbeiten, nutzten zahlreiche Konferenzteilnehmer die Möglichkeit, Kelsen, der zwar nicht an der Konferenz teilnahm ${ }^{58}$, diese aber doch mit großem Interesse von Berkeley aus verfolgte, ${ }^{59} \mathrm{zu}$ besuchen. Unter ihnen befand sich der Kolumbianer Luis E. Nieto Arteta. Wie Kelsen ihm zu verstehen gab $^{60}$ betrachtete Kelsen die egologische Auslegung Cossios als eigenständige Rechtstheorie, die mit der Reinen Rechtslehre nur oberflächliche Gemeinsamkeiten habe und lehnte sie daher im Hinblick auf die Reine Rechtslehre ab. Kelsens ablehnende Haltung der egologischen Interpretation dürfte auch Cossio über gemeinsame Bekannte wie Luis E. Nieto Areteta oder Antonio Sanchez de Bustamente y Montoro zu Ohren gekommen sein. ${ }^{61}$ Cossio jedoch war nach wie vor davon überzeugt, dass Kelsens Ablehnung einzig und alleine auf sprachlichen Missverständnissen und Verständigungschwierigkeiten beruhte. Um Kelsen von der egologischen Interpretation der Reinen Rechtslehre zu überzeugen und im persönlichem Dialog sprachliche Missverständnisse auszuräumen, versuchte Cossio seinen ehemaligen Schüler und Assisten-

\footnotetext{
${ }^{57}$ Otto Langfelder an Hans Kelsen, 3. 12. 1944, HKI, Nachlass Kelsen: 16b2.59.

58 MÉTALL, Kelsen 80.

${ }^{59}$ So berichtete der Argentinier Hugo Caminos, der 1945 als 24-Jähriger gemeinsam mit seinem Kollegen Ernesto Hermida bei Kelsen in Berkeley studiert hatte und für die Voice of America an der Konferenz von San Franciso teilnahm, jeden Abend Kelsen in seinem Haus besucht und ihm von den Geschehnissen auf der Konferenz „Bericht erstattet“ zu haben: Interview CAMINOS.

${ }^{60}$ NiETO ARTETA, Dialogo con el professor Kelsen.

${ }^{61}$ Cossio, Como ve Kelsen.
}

ten Ambrosio L. Gioja zu bewegen, zu Kelsen nach Berkeley zu reisen. ${ }^{62}$

Im Jahre 1945 kam über Rodolfo Bledel, den Kelsen gebeten hatte, "Society and Nature" ins Spanische zu übersetzen, der Kontakt Kelsens zum jungen argentinischen Juristen Jaime Perriaux zustande. Bledel, der den Übersetzungsauftrag von "Society and Nature" mit der Begründung ablehnte, nicht ausreichend mit der Materie des Werkes vertraut zu sein, empfahl Kelsen Jaime Perriaux als Übersetzer, zumal dieser mit "dieser Art von Übersetzungen mehr Erfahrung habe und auch schon Werke Ortega y Gassets ins Spanische übersetzt hätte." ${ }^{63}$ So übersetze der damals erst 25-jährige Argentinier Jaime Perriaux 1945 Kelsens "Society and Nature" ins Spanische. Im Herbst erhielt Perriaux ein Stipendium an der University of Michigan in Ann Arbor. Obwohl Perriaux das Studienjahr 1945/46 in den USA verbrachte, kam es vorerst zu keinem persönlichen Treffen mit Kelsen. Doch aufgrund seiner ausgezeichneten Englischkenntnisse konnte er Kelsens Werke ",aus erster Hand“ kennen lernen. Als Perriaux daher nach seinem Studienaufenthalt in den USA 1947 nach Argentinien zurückkehrte, wurde er zu einem der heftigsten Kritiker Cossios und warf ihm vor, die Reine Rechtslehre Kelsens zu missbrauchen. Gleiches gilt für die beiden jungen Argentinier Hugo Caminos und Ernesto Hermida, die im Sommersemester 1945 bei Kelsen in Berkeley studiert hatten. ${ }^{64}$

Inzwischen war in Argentinien 1946 Juan Peron an die Macht gekommen, der das Land mit Unterstützung des Militärs, der Arbeiterverbände und der Gewerkschaften autoritär regierte. Im Zuge der Regierung Perons kam es 1946 zu einer „Universitätsreform“ im Zuge deren Peron-

\footnotetext{
${ }^{62}$ GASSNER, OLECHOWSKI, Egologische Rechtslehre 10.

${ }^{63}$ Rodolfo Bledel an Hans Kelsen, 20.5. 1945, HKI, Nachlass Kelsen 16c1.59.

${ }^{64}$ Caminos an Kelsen, 24. 6. 1946, HKI Nachlass Kelsen 15s.58.
} 
kritische Universitätsangehörige sowie all jene, die sich weigerten, die Ideologie Perons in ihre Lehre einfließen zu lassen, aus den argentinischen Universitäten entfern wurden. ${ }^{65}$

Alleine in ihrer ersten Amtszeit ersetzte die Regierung Perons alle Richter des Obersten Gerichtshofes durch regimetreue Juristen und suspendierte ca. 1.500 regimekritische Hochschullehrer. Auch die Pressefreiheit wurde im Argentinien Perons stark eingeschränkt. Aufgrund seiner Nähe zum peronistischen Regime ${ }^{66}$ war Carlos Cossio 1946 zum Institutvorstand der Rechtsphilosophie an der Universität von Buenos Aires aufgestiegen, während sein ehemaliger Assistent Ambrosio L. Gioja zu jenen Universitätsangehörigen zählte, die sich weigerten, Perons Ideologie in ihre Lehre einfließen zu lassen und so ihre Stellen an der Universität verloren. Nunmehr als selbständiger Rechtsanwalt in Buenos Aires tätig, entschied sich Gioja Ende des Jahres 1947, dem Drängen Cossios nachzugeben und als „Bote der egologischen Rechtslehre" zu Kelsen nach Berkeley zu reisen, um diesen im persönlichen Gespräch von der „Richtigkeit der egologischen Interpretation der Reinen Rechtslehre" zu überzeugen. ${ }^{67}$ Mit „Empfehlungsschreiben" seines ehemaligen Kollegen Otto E. Langfelder ${ }^{68}$ - mit dem gemeinsam er 1944 ein Seminar über die Reine Rechtslehre und Cossios Ergänzungen gehalten hatte $^{69}$ - und seines Maestros Carlos Cossios ${ }^{70}$ ausgestattet, reiste Gioja in Begleitung seiner Ehefrau Elda im Sommer 1948 zu Kelsen nach

\footnotetext{
${ }^{65}$ So etwa: RiEKENBERG, Kleine Geschichte Argentiniens 56.

66 SARLO, Gira suramericana 404.

${ }^{67}$ GASSNER, OlECHOWSKI, Egologische Rechtslehre 10.

${ }^{68}$ Otto Langfelder an Hans Kelsen, 20. 12. 1947, HKI, Nachlass Kelsen 16b2.59.

${ }^{69}$ Otto Langfelder an Hans Kelsen, 3. 12. 1944, HKI, Nachlass Kelsen 16b2.59.

${ }^{70}$ Carlos Cossio an Hans Kelsen, 19. 12. 1947, HKI, Nachlass Kelsen 12IIg.52.
}

Berkeley.71 Bevor Gioja allerdings zu Kelsen nach Berkeley reiste, dürfte er im Juni 1948 Josef Kunz an der Universität von Toledo besucht haben. ${ }^{72}$

Kelsen dürfte Gioja bereits vor dessen Eintreffen in Berkeley zumindest vom „Hörensagen“ gekannt haben, zumal dieser zum Einen 1945 das Vorwort zur spanischen Ausgabe von "Peace through law"73 verfasst hatte und zum Anderen in Langfelders Briefen häufig erwähnt worden war. So hatte Langfelder etwa in einem Brief aus dem Jahre 1945 an Kelsen geschrieben, dass Gioja der einzige unter Cossios Schülern sei, der „[...] innerlich das Verhältnis Reine Rechtslehre und Teoria Egologica nicht ganz im Sinne von Altes Testament/Neues Testament ansehe. ${ }^{\text {“74 }}$ In einem kurz vor Giojas USA-Reise verfassten Brief an Kelsen bezeichnet Langfelder Gioja dann , $[\ldots]$ trotz einigen leichten Defekten, die an seinen skolastischen Anfängen hängen, als einen der tüchtigsten jüngeren Rechtsphilospohen Argentiniens. “75

Im Herbst 1948 besuchte Gioja zahlreiche Vorlesungen bei Kelsen in Berkeley und diskutierte nach eigenen Angaben mit Kelsen regelmäßig über die egologische Interpretation der Reinen Rechtslehre. ${ }^{76}$ In welcher Form diese Diskussionen allerdings wirklich stattfanden ist insofern

\footnotetext{
${ }^{71}$ Aus einem Brief des italienischen Rechtstheoretikers Elis Gianturcos, der Italien aufgrund des zweiten Weltkrieges verlassen musste und an der University of Chicago eine Anstellung als Lehrbeauftragter für italienische Literatur fand, sowie aus einen Brief Josef Kunz an Kelsen vom 25. 6. 1948 lässt sich erschließen, dass es erst frühestens im Juli 1948 erstmals zu einem persönlichem Treffen zwischen Kelsen und Gioja kam: HKI, Nachlass Kelsen 16c4.60.

72 Josef Kunz an Hans Kelsen, 25. 6. 1948, HKI, Nachlass Kelsen 16b1.59.

${ }^{73}$ Kelsen, Paz por medio del derecho.

${ }^{74}$ Otto Langfelder an Hans Kelsen, 6. 12. 1945, HKI, Nachlass Kelsen 16 b2.59.

${ }^{75}$ Otto Langfelder an Hans Kelsen, 21. 12. 1947, HKI, Nachlass Kelsen 16 b2.59.

${ }^{76}$ Gassner, OlechOWSKI, Egologische Rechtslehre 10.
} 
fraglich, zumal Gioja kaum Englisch und Kelsen wie bereits erwähnt kein Spanisch sprach. ${ }^{77}$ Während seines Aufenthalts bei Kelsen in Berkeley dürfte Gioja regelmäßig Cossio von seinen „Erfolgen“ in den Diskussionen mit Kelsen berichtet haben. So berichtet etwa Josef Kunz in einem Brief vom 8. November 1948 an Kelsen, von Cossio per Luftpost einen Brief erhalten zu haben, indem Letztgenannter von einem langem Brief Giojas aus Berkeley berichtet, in welchem Gioja stolz verkündete, Kelsen nun „vollständig egologisiert" zu haben. Kunz zufolge zitierte Cossio in seinem Schreiben wörtlich folgende Passage aus dem besagten Brief Giojas: „[...] Kelsen gibt zu, dass Cossios Interpretationen der Reinen Rechtslehre als bloß formale Logik, nicht Rechtswissenschaft, korrekt ist. Kelsen gibt zu, dass das Objekt der Rechtswissenschaft nicht Rechtsnormen sondern menschliches Verhalten ist. Kelsen sagt ferner, dass er die rationalen Aspekte bloß gegen den Rechtssoziologen betont hat. Kelsen stellt sich ganz auf den Boden Cossios egologischer Theorie." Cossio sei nun ganz aufgeregt und verlange eine offizielle Bestätigung von Kelsen, so Kunz in seinem Brief an Kelsen weiter. Wie aus dem Brief weiter hervorgeht, hielt Kunz persönlich Giojas Berichte an Cossio für ein Missverständnis, das wahrscheinlich durch Giojas mangelhaftes Englisch ausgelöst und durch die Tatsache, dass Gioja nur das hörte, was er hören wollte, verschlimmert wurde. ${ }^{78}$

Anscheinend nahm Kelsen auf das Schreiben Kunz' in einem Antwortschreiben vom 22. November 1948 Bezug, denn in seinem Brief vom 8. Dezember 1948 meint Kunz zu den Ausfüh-

\footnotetext{
77 So entschuldigt sich Gioja in einem Brief an Kelsen vom 27. 3. 1949, dass er erst jetzt schreibe, aber daran sei sein schlechtes Englisch schuld, dass es nötig mache immer einen Übersetzer zu haben, um sich an Kelsen wenden zu können: HKI, Nachlass Kelsen 15s.58.

${ }^{78}$ Josef Kunz an Hans Kelsen, 8. 11. 1948, HKI, Nachlass Kelsen 16b1.59.
}

rungen Kelsens hinsichtlich der Gespräche mit Gioja, dass er sich das genau so vorgestellt hätte. Gioja sei zwar ein reizender Mensch, dennoch sei im persönlichen Gespräch mit Gioja Vorsicht geboten. ${ }^{79}$

Vermutlich durch die Berichte Giojas aus Berkeley veranlasst, entwickelte Cossio im Laufe des Jahres 1948 eine regelrechte Obsession, Kelsen nach Argentinien zu holen und ihm dort ein Bekenntnis zur egologischen Interpretation der Reinen Rechtslehre abzugewinnen. ${ }^{80}$ In seinem am 31. Dezember 1948 in der argentinischen Zeitschrift La Ley veröffentlichten Aufsatz „Wie sieht Kelsen die egologische Rechtslehre?", also zu einem Zeitpunkt, als Kelsen sich nicht einmal noch zur Einladung Cossios offiziell geäußert hatte, kündigte Cossio bereits an, Kelsen werde im Sommer 1949, nach Argentinien kommen, um mit der egologische Rechtslehre in einen Dialog zu treten. ${ }^{81}$

Auch Kelsen war durch die Berichte seiner (argentinischen) Schüler und Freunde wie Josef Kunz, Jaime Perriaux oder Otto Langfelder von der eigenen Gestalt, die seine Reine Rechtslehre in Argentinien durch die Auslegung Carlos Cossios anzunehmen drohte, beunruhigt. So entschied er sich zu Beginn des Jahres 1949 nach Argentinien zu reisen und der dortigen Auslegung seiner Reinen Rechtslehre ein Ende zu setzen. ${ }^{82}$

\section{Kelsens Südamerikareise 1949 und das Nachspiel in den 50er Jahren}

Die Nachricht von Kelsens für den Sommer 1949 geplante Südamerikareise verbreitete sich nicht

\footnotetext{
${ }^{79}$ Josef Kunz an Hans Kelsen, 8. 12. 1948, HKI, Nachlass Kelsen 12IIg.52.

${ }^{80}$ Interview CAMINOS.

${ }^{81}$ Cossio, Como ve Kelsen.

${ }^{82}$ GASSNER, OleCHOWSKI, Egologische Rechtslehre 11.
} 
zuletzt aufgrund von Cossios Artikel „Wie sieht Kelsen die egologische Rechtslehre?" wie ein Lauffeuer am gesamten südamerikanischen Subkontinent. Ehemalige Kelsenschüler und Kelsenanhänger aus ganz Südamerika wandten sich an Kelsen und luden ihn ein, im Rahmen seiner Südamerikareise Vorträge an ihren Universitäten $\mathrm{zu}$ halten. Kelsen wollte allerdings vorerst nur den Einladungen aus Argentinien ${ }^{83}$ Folge leisten.

Zum Zeitpunkt von Kelsens Argentinienreise waren die rechtsphilosophischen Lager in Argentinien äußerst gespalten: Anhänger Carl Schmitts, Exil-Spanier, die wegen ihrer linken politischen Einstellung aus Spanien vertrieben worden waren, und natürlich eine große Zahl von Kelsen-Anhänger standen einander gegenüber. ${ }^{84}$ Die Kelsen-Anhänger ihrerseits spalteten sich einerseits in die Anhänger Cossios und seiner egologischen Rechtslehre und andererseits in die "orthodoxen" Kelsenianer, die dem an den argentinischen Universitäten eingeführten "Peronismus" kritisch gegenüberstanden und Cossio als einen Opportunisten des peronischen Systems betrachteten. ${ }^{85}$ Kurz vor Kelsens Argentinienreise im Sommer 1949 wurde die ohnehin schon angespannte Lage neuerlich angeheizt, zumal sich Cossios einstiger Freund, Schüler und Vertrauter Ambrosio L. Gioja von diesem lossagte und sich nunmehr auf die Seite der "orthodoxen Kelsianer" schlug. Hinzu kam außerdem, dass die Regierung Peron Kelsens Besuch in Argentinien zu eigenen Zwecken zu

\footnotetext{
${ }^{83}$ Kelsen wurde sowohl von der Rechtswissenschaftlichen Fakultät der Universität von Beuenos Aires, als auch vom Colegio de Abogados de Buenos Aires (einer der österreichischen Anwaltsakademie entsprechende Institution) und dem Instituto de Filosofia Juridica y Social, dem Cossio vorstand, nach Argentinien eingeladen. Siehe dazu auch SARLO, Gira sudamericana 408.

${ }^{84}$ SARLO, Gira sudamericana 413.

${ }^{85}$ Interview CAMINOS.
}

inszenieren versuchte, 86 was zur Folge hatte, dass einige von Kelsens Anhängern diesen vor einer Reise nach Argentinien warnten. ${ }^{87}$ So hatte sogar im argentinischen Parlament anlässlich vom Kelsens bevorstehenden Besuch eine heftige Debatte diesbezüglich stattgefunden. ${ }^{88}$ Noch wenige Tage vor seiner Abreise aus den USA ereilte Kelsen ein Brief seiner ehemaligen argentinischen Schüler Hugo Caminos und Ernesto Hermida, indem diese ihm dringend von seiner Argentinienreise abrieten, ${ }^{89}$ zumal er auf Einladung der Universität von Buenos Aires nach Argentinien reise und er somit per se zum Gast der argentinischen Regierung würde. ${ }^{90}$ Nichtsdestotrotz trat Kelsen, begleitet von seiner Gattin Grete, seine Argentinienreise an und schiffte sich am 15. Juli 1949 in New York auf der "SS Argentina“ der Schifffahrtsgesellschaft MooreMcCormack ein, die ihn über Trinidad, Rio de Janeiro, Sao Paulo und Montevideo, nach Buenos Aires bringen sollte. ${ }^{91}$ Gerüchten zufolge hatte ein ehemaliger Schüler Kelsens, der nunmehr für die Schifffahrtsgesellschaft MooreMcCormack arbeitete, Kelsen ein Ticket erster Klasse angeboten, was Kelsen aber dankend ablehnte. In Rio de Janeiro unterbrach Kelsen für einige Tage seine Reise, um seinen ehemaligen Schüler Hans Klinghoffer wiederzusehen. ${ }^{92}$

\footnotetext{
${ }^{86}$ So etwa war 1949 eine neue Verfassung erlassen worden, die wegen der zahlreichen weitgehenden Befugnisse, die sie dem Staatspräsidenten einräumte, zahlreiche Kritik erntete. Vgl. dazu RIEKENBERG, Kleine Geschichte Argentiniens 153.

${ }^{87}$ GASSNER, OLECHOWSKI, Egologische Rechtslehre 11.

${ }^{88}$ Metall, Kelsen 84; dies bestätigte auch im Interview CAMINOS.

${ }^{89}$ GASSNER, OlechOWSKI, Egologische Rechtslehre 11.

${ }^{90}$ Hugo Caminos und Ernesto Hermida an Hans Kelsen, 25. 6. 1949, HKI, Nachlass Kelsen 15s.58.

91 SARLO, Gira sudamericana 410.

92 Dies ergibt sich aus: Hans Klinghoffer an Hans Kelsen, 4. 8. 1949, HKI, Nachlass Kelsen, 15s.58; Darin schrieb Klinghoffer: „[...] Selbstverständlich rechne ich damit, dass Ihre Frau Gemahlin und Sie in der Ihnen schon bekannten Feiglschen Wohnung Quartier
} 
Klinghoffer veranlasste, dass Hans Kelsen und seine Gattin während ihres kurzen Aufenthalts in Rio de Janeiro in der Wohnung des aus Wien immigrierten Ehepaar Feigl in der Rua Gomes Carneiro 161 Unterkunft fanden. Während Kelsens Zwischenstopp in Rio de Janeiro dürfte ihn Klinghoffer von seinen Plänen, nach Israel auswandern $\mathrm{zu}$ wollen und von seiner Bewerbung an der neu gegründeten Hebräischen Universität von Jerusalem, berichtet haben, zumal Kelsen am 27. Juli 1949 aus Rio de Janeiro ein an den Dekan der neuen Fakultät in Jerusalem Nathan Feinberg gerichtetes Empfehlungsschreiben für Klinghoffer verfasste. ${ }^{93}$ Am 1. August lief das Linienschiff mit Hans und Grete Kelsen an Bord im Hafen von Montevideo ein, wo sie von Prof. Eduardo Couture ${ }^{94}$ von der Rechtswissenschaftlichen Fakultät von Montevideo bereits erwartet wurden. ${ }^{95}$ Aber auch Hugo Caminos und Ernesto Hermida waren nach Montevideo gekommen, um Kelsen vor der Weiterreise nach Argentinien nochmals persönlich zu warnen. Offensichtlich war Kelsens Argentinien-Reise in manchen uruguayischen Tageszeitungen angekündigt worden, zumal ein gewisser Guillermo (vormals Wilhelm) Kessler, der um 1926 bei Kelsen in Wien promoviert haben dürfte, am 1. August 1949 an Kelsen schrieb: ,[...] Soeben lese ich in der hiesigen Tageszeitung, dass Sie auf der ,Argentina', die heute hier anlegte, nach Buenos Aires fahren. Ich habe mich sofort mit dem Hafen in Verbin-

nehmen, ich werde dafür sorgen, dass Verpflegung, Bedienung usw. diesmal besser klappen."

${ }^{93}$ Empfehlungsschreiben Hans Kelsens vom 27.7. 1949 abgedruckt in: KLEIN, Klinghoffer 177.

${ }^{94}$ Eduardo J. Couture, Professor für Verfahrensrecht an der Universität von Montevideo und Rechtsanwalt in Montevideo, war ein großer Bewunderer Hans Kelsens und trug wesentlich zur Verbreitung von Kelsens Rechtslehre in Uruguay bei. Vermutlich nahm Eduardo J. Couture im Jahre 1941 ebenfalls an der Konferenz in Kuba teil und lernte so Hans Kelsen damals persönlich kennen: Interview SARLO.

${ }^{95}$ SARLO, Gira sudamericana 411. dung gesetzt, aber leider nur mehr erfahren, dass das Schiff schon wieder ausgelaufen ist. [...]." ${ }^{\prime 96}$

Kelsen und seine Gattin Grete verbrachten den Tag gemeinsam mit Couture, Caminos und Hermida in Montevideo, wo sie mit anderen uruguayschen Juristen zusammentrafen. Kelsen wurde gebeten, seinen Aufenthalt in Buenos Aires zu unterbrechen und am 15. August einen Vortrag an der Universität von Montevideo zu halten, was er auch zusagte. Bereits vor seiner Abreise aus den USA, hatte sich Werner Goldschmidt, Sohn des nach Montevideo immigrierten und 1940 dort verstorbenen deutschen Prozessrechtlers James Goldschmidt, der die „dreidimensionale Rechtslehre" Miguel Reales aus Brasilien „importiert" und in Argentinien salonfähig gemacht hatte und zu diesem Zeitpunkt in Argentinien an der Universidad Nacional de Tucuman internationales Privatrecht lehrte, in einem Brief an Kelsen gewandt und ihn im Namen seiner uruguayischen Freunde eingeladen, in Uruguay vorzutragen, ${ }^{97}$ doch dürfte Kelsen die Einladung damals ausgeschlagen haben.

Während eines gemeinsamen Mittagessens im Golfclub von Montevideo wurde Kelsen mit der Erstellung eines Rechtsgutachtens über das sich in uruguayischem Gewahrsam befindende italienische Kriegssschiff „Fausto" beauftragt98, ehe er am folgenden Tag, dem 2. August 1949, nach Buenos Aires weiterreiste, wo er am Hafen von Buenos Aires von einer Delegation der Universitäten von Buenos Aires und La Plata, der unter anderem neben Carlos Cossio, Ambrosio Gioja, Jaime Perriaux, Sebastian Soler auch Abel Aristegui und Roberto Vernengo angehörte, empfangen und in sein Hotel in der Florida 1005

\footnotetext{
${ }^{96}$ Guillermo (Wilhelm) Kessler an Hans Kelsen, 1. 8. 1949, HKI, Nachlass Kelsen, 15s.58.

${ }^{97}$ Werner Goldschmidt an Hans Kelsen vom 26. 6. 1949, HKI, Nachlass Kelsen, 15s.58.

98 Kelsen, Opinion Concerning the Ship Fausto.
} 
(heute Mariott Plaza) ${ }^{99}$ begleitet wurde. In Buenos Aires hielt Kelsen am 5., 10., 13. und 18. August an der Rechtswissenschaftlichen Fakultät von Buenos Aires vier Vorträge in französischer Sprache. Am 8. August hielt Kelsen am Instituto de Filosofia Juridica einen Vortrag über „Die Doktrin des Naturrechts vor dem Tribunal der Wissenschaften"100 und am 19. August trug Kelsen am Colegio de Abogadoa de Buenos Aires über „Kollektive und Individuelle Verantwortlichkeit für Staatsakte im Völkerrecht"101 vor. Am 22. August sprach Kelsen schließlich im Haus der oppositionellen Zeitung La Prensa über „Die NATO und die UN-Charta“.102 Auch leistete Kelsen der Einladung der nahe Buenos Aires gelegenen Universidad de La Plata folge, wo er am 23. August 1949 im Gebäude der kunstgeschichtlichen Universität einen Vortrag hielt. ${ }^{103}$ Abgesehen von der Universität von Buenos Aires und der von La Plata hatten zahlreiche andere argentinische Universitäten Kelsen zu Vorträgen eingeladen, deren Einladung Kelsen jedoch keine Folge leistete. Hervorzuheben ist die Einladung durch Robert Goldschmidt (Sohn von James und Bruder von Werner Goldschmidt) ${ }^{104}$ an die Universidad Nacional de Cordoba. In einem Brief von August 1949 schrieb er an Kelsen, wie sehr so-

\footnotetext{
${ }^{99}$ Interview CAMINOS.

${ }^{100}$ KELSEN, Natural Law Doctrine.

${ }^{101} \mathrm{KELSEN}_{L}$ Collective and Individual Responsability for.

102 KeLSEN, Atlantic Pact and UN Charta 10.

${ }^{103}$ Universidad de La Plata an Hans Kelsen, Dezember 1949, HKI, Nachlass Kelsen, 15s.58. Aus dem Schreiben geht auch hervor, dass Kelsen für seinen Vortrag 1.000 argentinische Pesos erhielt; auch die übrigen Vorträge dürften - wie damals üblich - fürstlich honoriert worden sein.

104 Wie aus einem Schreiben von Werner und Robert Kelsen an Hans Kelsen aus Anlass von dessen Argentinien-Besuch hervorgeht, lernten die beiden Brüder Kelsen (wahrscheinlich in den 30er Jahren) über ihren Vater in Berlin kennen, wo Kelsen die Goldschmidts auch in deren Haus in Berlin-Grünewald besucht haben dürfte.
}

wohl er selbst als auch seine (kranke) Mutter sich über ein Wiedersehen freuen würden. ${ }^{105}$

Der Besuch Kelsens in Argentinien wurde von der Diskussion um die richtige Auslegung der Reinen Rechtslehre überschattet, sodass Kelsen erleichtert zu sein schien, am 15. August Argentinien für ein paar Stunden verlassen zu können um per Flugzeug nach Montevideo zu reisen und dort an der Universität von Montevideo über ,die Doktrin des Naturrechts vor dem Tribunal der Wissenschaft" vorzutragen. ${ }^{106}$ Aber selbst in Uruguay entging Kelsen kritischen Fragen über seine Haltung zur egologischen Rechtslehre Cossios nicht. Die Eröffnungsrede zu seinem Vortrag wurde vom Institutsvorstand der Rechtsphilosophie Antonio M. Grompone gehalten, der mit Kritik an Kelsens Reiner Rechtslehre nicht sparte. ${ }^{107}$ Bei einem abschließenden Mittagessen antwortete Kelsen auf die Frage des Professors Eduardo Jimenez de Arechaga, wie Kelsen zu Cossios Lehre stehe, "[...] C'est pas de l' egologie, mais de l'egolatrie." 108 Anlässlich Kelsens Besuch in Montevideo publizierte Eduardo J. Couture eine Übersetzung Roberto Vernengos von Kelsens „Absolutism and Relativism in Philosophy and Politics" ${ }^{\prime 109}$.

In Argentinien konnte Kelsen keinen Vortrag abhalten, ohne dass er von Cossio coram publico zu einer Art Duell herausgefordert wurde, wobei Cossio jedes Mal überzeugt war, als Sieger aus diesen Diskussionen hervorgegangen zu sein. Cossio berichtete nach Kelsens Abreise, Kelsen habe der egologischen Rechtslehre (vor allem in privaten Unterhaltungen) wesentlich mehr Zugeständnisse gemacht, als er sich selbst eingestand. So habe er etwa seine aus Berkeley

\footnotetext{
${ }^{105}$ Robert Goldschmidt an Hans Kelsen, August 1949, in: HKI, Nachlass Kelsen, 15s.58. 106 SARLO, Gira sudamericana 412.

${ }^{107}$ Abgedruckt in: SARLO, Reception of the Pure Theory of Law 293.

108 Siehe Anm. 107.

${ }^{109}$ Kelsen, Absolutismo y Relativismo.
} 
fertig mitgebrachten Vorträge abgeändert, um in seinen Vorträgen der egologischen Rechtslehre so manches Zugeständnis zu machen. Auch habe sich Kelsen während seines Aufenthaltes in Argentinien, nicht wie sonst in der Rolle des Angreifers und Aufdeckers befunden, sondern in der des Angegriffenen. ${ }^{110}$

In der Kontroverse zwischen Cossio und Kelsen ging es hauptsächlich darum, wer über die "richtige Anschauung der Reine Rechtslehre" verfügte. So schrieb Kelsen etwa: „Prof. Cossio tritt mit dem erstaunlichen Anspruch auf, dass die Vorstellung, die die Egologische Theorie von der Reinen Rechtslehre besitzt, die richtige und die Vorstellung die Kelsen von der Reinen Rechtslehre besitzt die falsche sei."111 Dieser Anspruch sei schon deshalb abzulehnen, da er der Autor der Reinen Rechtslehre sei und er wohl oder übel wisse, was er wie gemeint habe, als er die Reine Rechtslehre schrieb.112 Cossio seinerseits verwies insbesondere darauf, dass es gerade das Grundmerkmal einer wissenschaftlichen Theorie sei, dass diese von der Auslegung ihres Schöpfers verschieden interpretierbar ist. So soll Cossio Kelsen in einem persönlichem Gespräch anlässlich eines gemeinsamen Abendessen in Buenos Aires, bei welchem Kelsen Cossio sagte, er sei der Autor der Reinen Rechtslehre und wisse daher gut was er wie gemeint habe, erwidert haben: „Dr. Kelsen, Ihnen passiert dasselbe wie Kolumbus, dieser glaubte auch Indien entdeckt zu haben und war in Wirklichkeit in Amerika gelandet." 113 Wie zahlreiche Zeitzeugen ${ }^{114}$ berichteten, erreichte der "Streit“

\footnotetext{
${ }^{110} \mathrm{COSSIO}_{L}$ Egologische Theorie und Reine Rechtslehre 26.

${ }^{111}$ KELSEN, Reine Rechtslehre und Egologische Theorie 453.

112 Interview ARISTEGUI; Aristegui war Zeitzeuge bei den Gesprächen zwischen Cossio und Kelsen im Sommer 1949 in Argentinien.

${ }^{113}$ Interview ARISTEGUI.

114 So etwa: Interviews VERNENGO, ARISTEGUI, CAMINOS.
}

um die richtige Auslegung der Reinen Rechtslehre wenige Tage vor Kelsens Abreise aus Buenos Aires ihren Höhepunkt: So soll Kelsen nach einem Vortrag von Cossios Schüler, dem damals erst 25-jährigen José Vilanova, auf ein ständiges Nachfragen Cossios über Kelsens Auffassung hinsichtlich des Logikbegriffs ein „Eh bien, mon vieux, va faire l'amour!" entkommen sein. ${ }^{115}$

Abgesehen von den Streitigkeiten mit Carlos Cossio nutzte Kelsen seinen Argentinienaufenthalt, um langjährige Freunde, wie Otto E. Langfelder oder etwa seinen Jugendfreund Fritz Schey, der aufgrund des 2. Weltkrieges nach Argentinien emigriert war, wiederzusehen. ${ }^{116}$ Auch konnte Kelsen Kollegen, mit denen er schon seit Jahren in engem brieflichen Kontakt stand, wie etwa Jaime Perriaux, erstmals persönlich treffen. Perriaux schrieb anlässlich Kelsens Besuch in Argentinien, dass Kelsen in seinen Konferenzen, für all jene, die ihn kannten, nichts wirklich Neues gesagt habe. Da aber der Großteil der Rechtsgelehrten in Argentinien Kelsen nur über die Anhänger der egologischen Rechtslehre kennen gelernt hatten und somit nur die bereits von der Egologie beeinflusste Rechtslehre Kelsens kannten, brachten die Vorträge Kelsens insofern sehr wohl eine neue Sichtweise zutage, als sich für viele nicht deutschsprachige Argentinier zum ersten Mal die Möglichkeit bot, Kelsens Rechtslehre aus erster Hand kennen zu lernen. ${ }^{117}$

Als Kelsen am 25. August 1949 gemeinsam mit seiner Gattin zum Flughafen von Buenos Aires fuhr, um mit dem Morgenflug der argentinischen Fluggesellschaft FAMA nach Rio de Janeiro zu reisen, ${ }^{118}$ wurde ihm - im Gegensatz

\footnotetext{
115 SARLO, Gira sudamericana 413. Aristegui, der bei diesem Gespräch zugegen war, bestätigte dies im genannten Interview.

${ }^{116}$ Fritz Schey an Hans Kelsen, 18. 8. 1949, HKI, Nachlass Kelsen, 15s.58.

117 Perriaux, Balance cientifico 1.

118 SARLO, Gira sudamericana 414f.
} 
zu seiner Ankunft - kein großer Abschied bereitet. Die argentinische Delegation, die Kelsen am Flughafen offiziell verabschieden hätte sollen und der auch Cossio angehörte, war (angeblich) im Stau steckengeblieben und schaffte es so nicht rechtzeitig, zum Flughafen zu kommen. ${ }^{119}$ In Rio de Janeiro, wo Kelsen und seine Gattin vom 25. August bis zum 1. September 1949 weilten, bezogen die Kelsens abermals in der Wohnung der Feigls Quartier. Die Einladung Kelsens nach Rio de Janeiro erfolgte einerseits vom brasilianischen Rechtswissenschaftler und Politiker Prof. Bilac Pinto, der zu dieser Zeit an der Universidade do Brasil (nunmehr UFRJ) Verwaltungsrecht lehrte und andererseits von der Fundação Getulio Vargas, ${ }^{120}$ wo Kelsen am 29. August 1949 seinen Vortrag über „Die NATO und die UN-Charta" wiederholte. Am 31. August 1949 hielt Kelsen an der Universidad do Brasil einen weiteren Vortrag und im Anschluss daran entschied der Universitätssenat, Hans Kelsen eine Ehrenprofessur der Universidad do Brasil (Prof. honoris causa) zu verleihen, die er schließlich am 26. Juni 1952 erhielt, wobei Kelsen bei der offiziellen Verleihungszeremonie nicht zugegen war. Das Verleihungsdekret wurde vom österreichischen Botschafter stellvertretend für Hans Kelsen entgegengenommen. ${ }^{121}$ Während seines Aufenthaltes in Rio de Janeiro lernte Hans Kelsen auch den brasilianischen Rechtsphilosophen und Begründer der Dreidimensionalen Rechtslehre Miguel Reale, damals Professor an der Universidad de São Paulo, kennen. Über das Verhältnis der beiden ist je-

\footnotetext{
${ }^{119}$ Interview CAMINOS.

${ }^{120}$ DrumMOND, Kelsen in Rio de Janeiro. An dieser Stelle sei Dr. Thiago Thannous für seine zahlreichen inspirierenden Hinweise, sowie Filipe Drummond, M.A. aufrichtig gedankt.

${ }^{121}$ Unrichtig daher die Angaben von JEstAedt, Hans Kelsen Werke 1, 102, der den 25. 6. 1949 als Datum nennt; und von SARLO, Gira sudamericana 415, der von der Verleihung eines Ehrendoktorats am 31. August 1949 schreibt.
}

doch nichts bekannt. Am 1. September 1949 kehrten Kelsen und seine Gattin Grete per Schiff nach New York zurück.

Nach Kelsens Abreise verfasste Cossio einen Aufsatz, ${ }^{122}$ indem er über Kelsens Argentinienaufenthalt Bilanz zog. Dieser Aufsatz wurde von Otto E. Langfelder ins Deutsche übersetzt und erschien 1953 in der von Kelsen begründeten und nunmehr von seinem ehemaligen Schüler Alfred Verdross herausgegebenen Zeitschrift für Öffentliches Recht (ZÖR). ${ }^{123}$ Im Zusammenhang mit der Publikation von Cossios Artikel versicherte sich Verdross im Vorfeld bei Kelsen, ob dieser gegen eine Veröffentlichung von Cossios Artikel in der ZÖR etwas einzuwenden habe. ${ }^{124}$ Kelsen erwiderte, er freue sich auf das Erscheinen von Cossios Beitrag , ,[...] in der exzellenten Übersetzung von Otto E. Langfelder “"125, behalte sich aber das Recht auf eine Gegendarstellung vor. Die Gegenäußerung Kelsens erschien schließlich 1953 ebenfalls in der ZÖR, ${ }^{126}$ wozu sich Cossio im Jahre 1954 in La Ley bzw. in deutscher Version in der ZÖR 1956 abermals äußerte. ${ }^{127}$ Währenddessen hatte Carlos Cossio noch 1952 im Guillermo Kraft Verlag Buenos Aires die von Kelsen während seines Argentinienaufenthaltes 1949 gehaltenen Vorträge, ergänzt um die angeblich zwischen ihm und Cossio geführten Dialoge, unter dem Namen Kelsen - Cossio veröffentlicht. ${ }^{128}$ Als Kelsen über seine spanisch-sprachigen Schüler, allen voran über Josef Kunz und Jaime Perriaux, von der Existenz dieses Buches erfuhr, schrieb er am 30. Novem-

\footnotetext{
122 Cossio, Teoria egologica y teoria pura.

${ }^{123}$ Cossio, Egologische Theorie und Reine Rechtslehre $15 \mathrm{ff}$.

${ }^{124}$ Die Korrespondenz befindet sich in: HKI, Nachlass Kelsen, 16 c2.59.

125 Ebd. Siehe Anm. 125.

${ }^{126}$ KELSEN, Reine Rechtslehre und Egologische Theorie $449 \mathrm{ff}$.

${ }^{127}$ Cossıo, Polemica anti-egologica; Cossıo, Antiegologische Polemik.

${ }^{128}$ Kelsen, Cossio, Problemas escogidos.
} 
ber 1952 an die Zeitschrift La Ley und bat, sein Schreiben, in welchem er erklärte hatte, dass das Buch Kelsen - Cossio ohne seine Zustimmung und sein Zutun verfasst und veröffentlicht worden war, zu veröffentlichen. Gleichzeitig forderte er den Verlag Kraft auf, die bereits gedruckten Auflagen des Werkes Kelsen - Cossio einzuziehen, was schließlich im Sommer 1953 auch geschah. ${ }^{129}$ Im Streit um die nicht autorisierte Veröffentlichung von Kelsens in Argentinien gehaltenen Vorträgen sowie im Hinblick auf die Veröffentlichung von Cossios vorläufiger Bilanz von Kelsens Besuch in Argentinien, kam insbesondere Kelsens ehemaligem Schüler Otto E. Langfelder, der in Buenos Aires lebte und Cossios Aufsätze ins Deutsche übersetzte, eine wichtige Rolle zu, zumal er Kelsen in vertraulichen Briefen immer schon vorab über den Inhalt von Cossios Aufsätze informierte ${ }^{130}$ und es Kelsen so ermöglichte, dass dieser sich bereits vor offiziellem Erscheinen der Aufsätze mit einer Gegendarstellung beschäftigen konnte. Über einem direkten Kontakt zwischen Kelsen und Cossio nach dem Jahre 1952 ist nichts bekannt, ein solcher kann aufgrund der eben dargelegten Vorfälle jedoch nahezu ausgeschlossen werden.

Im Winter- und Sommersemester 1950/51 trat der damals 70-jährige Hans Kelsen einen Teil seiner Vorlesungen in Berkeley an Josef L. Kunz ab; am 25. April 1952 trat er in den Ruhestand und konnte mithilfe einer Subvention der Rockefeller Stiftung der Einladung des Institut Universitaire des Hautes Etudes International nach Genf folgen. ${ }^{131}$ So kehrte er für das Studienjahr 1952/53 nach Genf zurück und nahm die beiden Argentinier Ambrosio L. Gioja und Roberto Vernengo mit ans Institut Universitaire des Hautes Etudes International. Während seines Genf-

\footnotetext{
${ }^{129}$ Guillermo Kraft Ltda an Hans Kelsen, 16. 6. 1953, HKI, Nachlass Kelsen 16 c2.59.

${ }^{130}$ Otto Langfelder an Hans Kelsen, 7. 10. 1949, HKI

Wien Nachlass Kelsen 15s.58.

${ }^{131}$ MÉTALL, Kelsen 85.
}

Aufenthalts galt Kelsens ganze Aufmerksamkeit seinen Untersuchungen über die (juristische) Logik: 1951 war Georg Henrik von Wrights Werk „Deontische Logik" erschienen und Kelsen machte dieses Werk zum Gegenstand eines Privatseminars, an dem nur er, Gioja und Vernengo teilnahmen. ${ }^{132}$

\section{Kelsens Mexikoreise und der Vorschlag für den Friedensnobelpreis}

Parallel zu den vorhin geschilderten Kontakten mit den Juristen der La Plata-Staaten hatte Kelsen auch weiter seine Kontakte zu Mexiko, das er ja schon 1943 besuchen hätte sollen, gepflegt, und noch bevor er jemals dorthin gereist war, am 27. August 1951, hatte die Universidad Nacional Autónoma de México entschieden, Hans Kelsen ein Ehrendoktorat zu verleihen. ${ }^{133}$ Dies dürfte vor allem auf die beiden Professoren der UNAM Guillermo Hector Rodriguez und Emilio O. Rabasa zurückzuführen sein, die Kelsen so wie auch Guillermo Floris Margadant - in Berkeley besuchten. ${ }^{134}$ Emilio O. Rebasa dürfte neben Kelsens altem Bekannten Garcia Maynez auch federführend daran beteiligt gewesen sein, dass Kelsen im März/April 1960, damals 79 Jahre alt, auf Einladung der UNAM eine Woche lang Mexiko D.F. besuchte und als Gastprofessor Vorträge an der UNAM hielt. Die Rolle, die Kelsens ehemaliger Schüler Recenses Siches bei Kelsens Besuch in Mexiko spielte, ist nicht ganz klar: Anzunehmen ist, dass - da Recenses Siches Kelsen und Kunz zufolge den „Fehler" begangen hatte, in die Metaphysik abzudriften und zudem im Konflikt um die „richtige Auslegung

\footnotetext{
132 Interview VERNENGO.

${ }^{133}$ Rolando Tamayo y Salmoran behauptet hingegen, das Ehrendoktorat wurde am 21.6. 1951 verliehen: FLORES, Una visita a Hans Kelsen 59 Anm 6. ${ }^{134}$ FLORES, Una visita a Hans Kelsen 64.
} 
der Reinen Rechtslehre" Partei für Carlos Cossio und dessen egologische Rechtslehre ergriffen hatte - die Beziehung zwischen letztgenannten und Kelsen 1960 deutlich abgekühlt und distanziert war. Zwar war Recenses Siches Teil der Delegation, die Kelsen und seine Gattin Grete am 29. März 1960 am Flughafen von Mexico City abholten, doch berichtet der spätere UNAM-Professor und damalige Student Ulises Schmill Ordóñez, dass das Verhältnis der beiden ,"äußerst gespannt“ war. ${ }^{135}$ Im Vordergrund von Kelsens Mexiko-Besuch stand dessen 1960 in Mexiko erschienenes Werk „Einführung in die Reine Rechtslehre“, ${ }^{\text {“ }} 36$ zu welchem Emilio O. Rabasa auch das Vorwort verfasst hatte. Auch die vier an der UNAM anberaumten Vorträge, von denen Kelsen lediglich zwei hielt, da er schon nach wenigen Tagen an der "Rache des Montezuma“ erkrankte, ${ }^{137}$ sollten sich mit diesem Werk beschäftigen.

Kelsens Mexiko-Besuch wurde in allen großen mexikanischen Tageszeitungen (wie beispielsweise der "Novedades“) angekündigt, weshalb die Hörsäle, in denen Kelsen am 30. März und am 2. April 1960 seine Vorträge hielt, sprichwörtlich aus allen Nähten platzten. ${ }^{138}$ Kelsen, der in Mexiko im Hotel Presidente (heute Hotel InterContinental) residierte, stattete am 2. April 1960 - begleitet von Emilio O. Rabasa, Leiter der Doktoratstudiums an der UNAM, Riccardo Garcia Villalobos, Dekan der juristischen Fakultät der UNAM und Nabos Carillo, Rektor der UNAM - dem damaligen mexikanischen Staatspräsidenten Adolfo Lopez Mateos in dessen Amtsräumlichkeiten in Los Pinas einen Besuch ab, wobei sich Kelsen und Mateos, Zeitungsberichten zufolge, 139 über die "guten Beziehungen zwischen Österreich und Mexiko" unterhielten.

\footnotetext{
135 Interview SCHMILL ORDÓÑEZ.

${ }^{136}$ KeLSEN, Introducción a la Teoria Pura del Derecho.

${ }^{137}$ Interview SCHMILL ORDÓÑEZ.

${ }^{138}$ FLORES, Una visita a Hans Kelsen 59.

139 Zeitung „Novedades“ vom 3. April 1960, 2.
}

Während seines Mexiko-Aufenthaltes wurde Kelsen nicht nur zum Ehrenmitglied der Sociedad Mexicana de Filosofia, sondern auch von deren Vorsitzenden José Luis Curiel im Namen der Gesellschaft auch für den Friedensnobelpreis vorgeschlagen - ein Vorschlag, dem bekanntlich jedoch keine Folge geleistet wurde. ${ }^{140}$

\section{Nachwort}

Bis heute scheint die Popularität Kelsens in Lateinamerika ungebrochen, und die Zahl seiner Anhänger ist kaum geringer als jene, die er heute in Europa hat. Die Reine Rechtslehre ist an vielen Universitäten fixer Bestandteil des juristischen Studiums, und noch immer finden Nachdrucke und Übersetzungen seiner Werke ins Spanische und Portugiesische statt. Dies steht in einem denkwürdigen Kontrast zu den USA, wo Kelsen immerhin zwölf Jahre lang unterrichtete und bis zu seinem Tod 1973 lebte, und wo seine Lehre kaum Widerhall gefunden hat.

Die Gründe dafür sind vielfältig. Hinzuweisen ist zunächst auf die unterschiedlichen Rechtssysteme; denn wenn auch Kelsen mit dem Anspruch aufgetreten war, eine Theorie des positiven Rechts „schlechthin“ zu verfassen, ${ }^{141}$ also eine Theorie, die auf jedes Rechtssystem anwendbar sei, so war doch sein ganzes Denken vom kontinentaleuropäischen System mit dem Vorrang des Gesetzesrechtes geprägt und ist etwa der Stufenbau der Rechtsordnung viel leichter einem Juristen zu erklären, der es gewohnt ist, mit einer geschriebenen Verfassung, mit vom Parlament erlassenen Gesetzen und von Ministern erlassenen Verordnungen zu arbeiten, als einem Juristen, der vorwiegend mit Richterrecht arbeitet. Hinzuweisen ist hier auf den beinahe schon vergessenen Umstand, dass

${ }^{140}$ Flores, Una visita a Hans Kelsen 60 f; Metall, Kelsen 90.

${ }^{141}$ KELSEN, Reine Rechtslehre ${ }^{1} 1$. 
die juristischen Kontakte zwischen Österreich und Lateinamerika seit jeher eng waren, und sowohl das österreichische Strafgesetzbuch 1803 als auch das Allgemeine bürgerliche Gesetzbuch 1811 vorbildhaft für eine Reihe lateinamerikanischer Kodifikationen waren. ${ }^{142}$ So fielen die Lehren Kelsens in Lateinamerika auf fruchtbaren Boden.

Dann aber, und dies haben die vorstehenden Ausführungen gezeigt, waren es vielfach die persönlichen Kontakte und somit auch Zufälligkeiten, die Kelsens Lehre in Lateinamerika populär machten. Hatten vor 1930 einige lateinamerikanische Studierende bei Kelsen in Wien studiert, so waren es später die Kelsen-Schüler Métall, Klinghoffer, Kunz und Langfelder, die vor der NS-Verfolgung nach Südamerika flohen und hier für die Verbreitung der Reinen Rechtslehre sorgten. Eine Schlüsselrolle aber kam sicherlich Carlos Cossio zu. Seine „egologische Rechtslehre" hatte zwar - bei näherer Betrachtung - mit der Reinen Rechtslehre nur sehr wenig zu tun, berief sich aber auf sie und trug so wesentlich zur Popularität auch der Reinen Rechtslehre in Argentinien bei. Bemerkenswerter Weise überdauerte diese bis heute, während die egologische Rechtslehre selbst heute kaum noch gelehrt wird. So verdankt Kelsen seinem Kontrahenten Cossio vielleicht mehr, als ihm selbst jemals bewusst wurde.

142 BRAVo LiRA, Codigo penal de Austria 314.

\section{Korrespondenz:}

Dr. Miriam Gassner

Seilerstätte 28, 1010 Wien, Österreich

miriam.gassner@univie.ac.at
Literatur:

Nicoletta BERSIER LADAVAC, Rudolf Aladár Métall, in: WALter, JABLONER, ZelENY, Kreis um Hans Kelsen 315-324.

Bernardino BRAVO LIRA, El código penal de Austria (1803) epicentro de la codificación penal en tres continentes, Anuario de Filosofía Jurídica y Social (Valparaíso 2003) 299-344.

Emilio Fernández CAMUS, Filosofia juridica contemporanes (La Habanna 1932).

Simon COllier, William F. SATER, A History of Chile 1808-1994 (Cambridge-New York-Melbourne 1996).

Carlos Cossio, La Reforma Universitaria o el Problema de la Nueva Generación (Buenos Aires 1927).

Carlos Cossio, El concepto puro de la revolution (Barcelona 1936).

Carlos Cossio, El jurista de la epoca contemporanea, in: „La Ley“ vom 12. 101941.

Carlos Cossio, Prólogo in: Hans Kelsen, La Teoria Pura del Derecho (Buenos Aires 1941) 7-15.

Carlos Cossio, La teoría egológica del derecho y el concepto jurídico de libertad (Buenos Aires 1944).

Carlos Cossio, Como ve Kelsen a la Teoria Egologica del Derecho? in: „La Ley“ vom 31. 12. 1948, 1-3.

Carlos Cossio, Teoria egologica y teoria pura Balance provisional de la visita de Kelsen a la Argentina in: „La Ley“ vom 25. 10. 1949.

Carlos Cossio, Egologische Theorie und Reine Rechtslehre. Eine vorläufige Bilanz von Kelsens Besuch in Argentinien, in: ZÖR NF 5 (1953) 15-69.

Carlos Cossio, La polemica anti-egologica (respuesta al professor Hans Kelsen), in: „La Ley“ vom 7. 10. 1954, 1-6. 
Carlos Cossio, Die anti-egologische Polemik (Erwiderung an Professor Kelsen) in: ZÖR NF 8 (1957/58) 189-231.

Filipe DRUMMOND, Kelsen in Rio de Janeiro (unveröffentlichter Recherchebericht vom 15. März 2012).

Immer B. Flores, Una visita a Hans Kelsen a Mexico, Anuario de Filosofia y Teoria del Derecho 8 (2014) 53-94.

Eduardo GARCíA MAYNEZ, Introducción a la lógica jurídica (México 1951).

Miriam Gassner, Thomas OlechOWSKI, Egologische Rechtslehre versus Reine Rechtslehre. Cossio versus Kelsen, in: Rechtstheorie 44 (2013) 139-156.

Matthias JestAedT, Hans Kelsen Werke, Bd. 1 (Tübingen 2007).

Jörg KAMMERHOFER, Josef Laurenz Kunz, in: WALTER, JABLONER, ZELENY, Kreis um Kelsen 243-259.

Hans KELSEN, Bemerkungen zur Chilenischen Verfassung in: ZÖR 5 (1926) 616-619.

Hans Kelsen, Compendio esquemático de una teoría general del Estado (Barcelona 11928, 21934).

Hans Kelsen, La Teoria Pura del Derecho. Método y Conceptos Fundamentales (Madrid 1933).

Hans Kelsen, A competência da Assembléia Nacional Constituinte, in: Politica. Revista de Direito Público, Legislaçao Social e Economia 1 (1934) 3543.

Hans KELSEN, Reine Rechtslehre (Leipzig-Wien ${ }^{1} 1934$ ).

Hans Kelsen, Teoría general del Estado (BarcelonaMadrid-Buenos Aires 1934).

Hans Kelsen, La teoria pura del derecho y la jurisprudencia analitica, in: „La Ley" vom Dezember 1941, 197-208.

Hans Kelsen, The Pure Theory of Law and Analytical Jurisprudence, in: Harvard Law Review 55 (1941) 44-70.

Hans KelSEN, La paz por medio del derecho (Buenos Aires 1946).

Hans Kelsen, Collective and Individual Responsability for Acts of State in International Law in: Jewish Yearbook of International Law (1948/49) 226-239.

Hans KeLSEN, El absolutismo y el relativismo en la filosofia y en la politica, in: Zeitung „La Ley“ vom 2. 8. 1949, 1-3.

Hans Kelsen, The Atlantic Pact and the UN Charta in: The New Leader 32/10 (1949) 10.

Hans Kelsen, The Natural Law Doctrine before the Tribunal of Science in: Western Political Quarterly 2 (1949) 481-513.

Hans KELSEN, Reine Rechtslehre und Egologische Theorie. Antwort auf: Carlos Cossio, Egologische Theorie und Reine Rechtslehre, eine vorläufige Bi- lanz von Kelsens Besuch in Argentinien, ZÖR NF 5 (1953) 449-482, 453.

Hans Kelsen, Carlos Cossio, Problemas escogidos de la Teoria Pura del Derecho (Buenos Aires 1952).

Hans Kelsen, Introducción a la Teoria Pura del Derecho (Mexico 1960).

Hans Kelsen, Opinion Concerning the Claims of the Italian Owners of the Ship Fausto, in: ZÖR NF 37 (1986/1987) 1-15.

Claude KleIN, Hans (Itzhak) Klinghoffer, in: WALTER, JABLONER, ZELENY, Kreis um Hans Kelsen 175-184.

Josef L. KUNZ, Latin-American philosophy of law in the twentieth century (New York 1950)

Luis LEGAS y LACAMBRA, Kelsen. Estudio crítico de la teoría pura del derecho y del estado de la Escuela de Viena (Barcelona 1933).

Uwe LÜBKEN, Bedrohliche Nähe. Die USA und die nationalsozialistische Herausforderung in Lateinamerika 1937-1945 (Stuttgart 2004).

Rudolf A. MÉTALL, Hans Kelsen Leben und Werk (Wien 1969).

Rudolf A. MÉTALL, Hans Kelsen y su escuela vienesa de teoria juridica, in: Revista de Ciencias Sociales 6 (1974) 21-28.

Luis E. Nieto ARTETA, Un dialogo con el professor Kelsen sobre logica juridica in: Revista Universidad Nacional de Colombia 3 (1945) 11-31.

Gabriel NogueIRA DiAs, Luis Recaséns Siches, in: WALter, JABLONER, ZelenY, Kreis um Hans Kelsen 366-393.

Gregorio ROBLES MORCHON, Luis Legaz y Lacambra in: WALTER, JABLONER, ZELENY, Kreis um Hans Kelsen 261-291.

Jaime PERRIAUX, Balance cientifico de la visita de Kelsen, in: „La Ley“ vom 14. 09. 1949, 1.

Carlos PetToruti, Verbindung zwischen Wien und La Plata. Überlegungen über den Einfluss der Reinen Rechtslehre im Unterrichtswesen der Rechtstheorie an der Universität La Plata, in: WALTER, JABLONER, ZELENY, Hans Kelsen anderswo 227-241.

Michael RieKenberg, Kleine Geschichte Argentiniens (München 2009).

Hector RODRIGUEZ EsPINOZA, Quien fue Eduardo Garcia Maynez?

http://www.hectorrodriguezespinoza.com/noticias .php?categoria=110 (abgerufen am 08. 08. 2013).

Oscar SARLO, La gira sudamericana de Hans Kelsen. El frente sur de le Teoria Pura del Derecho, in: Ambiente Juridico 12 (2010) 400-425.

Oscar SARLO, The reception of the Pure Theory of Law in Uruguay (and the refutation of its alleged falsa- 
tion), in: WALter, JAblONER, ZelenY, Hans Kelsen anderswo 281-309.

Robert WALter, Clemens JABLONER, Klaus ZelenY, Einleitung, in: Robert WALTER, Clemens JABLONER, Klaus Zeleny (Hgg.), Der Kreis um Hans Kelsen. Die Anfangsjahre der Reinen Rechtslehre (= Schriftenreihe des Hans Kelsen-Instituts 30, Wien 2008) 1-23.

Robert Walter, Clemens JABloner, Klaus ZelenY (Hgg.), Hans Kelsen anderswo. Hans Kelsen abroad. Der Einfluss der reinen Rechtslehre auf die Rechtstheorie in verschiedenen Ländern, Teil III (= Schriftenreihe des Hans Kelsen-Instituts 33, Wien 2010) 227-241.

\section{Interviews:}

Hugo CAminos, 31. 3. 2012, Buenos Aires. Oscar SARLO, 23. 3. 2012, Montevideo.

Abel Javier ARISTEGUI, 28. 3. 2012, La Plata.

Roberto VERNENGO, 26. 3. 2012, Buenos Aires.

Ulises SCHMILl ORdóÑEZ, 8. 12. 2012, Mexico City. 